\title{
Dynamic Analysis of Innovative Hybrid Wind Mill Tower with Soil Structure Interaction
}

\author{
Hemal J. Shah and Atul K. Desai
}

\begin{abstract}
The wind mill towers are constructed using monopoles or lattice type tower. As the height of tower increases it gives more power but it becomes uneconomical, So in this research work innovative hybrid wind mill tower such as combination of monopole and lattice tower is analyzed using FEM software. When the tall structures are constructed on soft soil it becomes dynamically sensitive so three types of soil such as hard, medium and soft are also considered and the frequencies of innovative hybrid tower are studied for different types of soil. From study it is revealed that the innovative hybrid tower will reduce resonance condition considering soil structure interaction.
\end{abstract}

Index Terms - Wind mill tower, dynamic analysis, Resonance conditions, soil structure interaction.

\section{INTRODUCTION}

From the mid part of the 19th century to till date, fossil fuels have provided the power necessary to complete many of society's most basic tasks worldwide. But in the recent years the renewable sources of energy have become most popular and there is more advancement in the technology.

One of the important sources of power generation is wind mills. According to details available from Government of India, the states like Gujarat, Karnataka, Kerala have utilized only 3 to 5 percent of their wind power potential. All states of India have total wind power utilization of only $26777 \mathrm{MW}$ which is only 8.86 percentage of the total wind power potential available in major states of India. The wide gap between the installed capacity and the assessed potential in India clearly indicates the opportunity in this field. So to utilize all available wind power potential there must have more advancement in the Wind Mill Technology. The height of the wind mills is increased in the recent years to extract more power at higher elevations. Basically, two types of tower system such as monopoles or lattice towers are used for the wind mill supporting towers. Each type of tower has its own advantages and disadvantages. As the height of the wind mill increases the thickness of the wall of the monopole towers is increasing and it untimely leads to increase in cost and uneconomical sections. On the other hand the lattice towers are formed by connecting the various angle or box sections by doing proper riveting at the site. The lattice towers will resist the loads by truss action of the members so members of towers are subjected to axial forces only. As the

Manuscript received December 10, 2017; revised May 1, 2018.

Hemal J. Shah is with the Government Engineering College, Bharuch, Gujarat, India (e-mail: hemal3007@gmail.com).

Atul K. Desai is with Sardar Vallabhbhai National Institute of Technology, Surat, Gujarat, India (e-mail: atuldesai61@gmail.com). lattice towers are open, wind will pass between the members and wind loads are reduced significantly on the towers.

The monopoles are used for lesser heights of the turbine while the Lattice tower can be used for turbines having lesser mass. If we increase the height of turbine it becomes single degree of freedom system and becomes dynamically sensitive particularly in soft soils. So in this study an innovative hybrid tower which is combination of monopole and lattice tower is proposed and the loads of earlier research works are applied on the innovative hybrid tower and the dynamic analysis of the tower is carried out considering 3 different types of soil.

\section{LITERATURE REVIEW}

Gencturk [1] has studied the various bracing system for 24 meter high lattice tower and various design alternatives are given for the wind mill lattice tower. Song [2] has studied the effects of the different earthquake on tall wind turbines and has concluded that dynamic response of structure depends on height. When the height increases to $177 \%$, the maximum displacement in the top of the tower would increase to $231 \%$ in 8-degree rare earthquake. Lombardi [3] has experimentally studied the effects of the soil structure interaction on the wind mills and has concluded that the clayey soils will make the tall structure dynamically sensitive. Hani [4] has done optimization of $100 \mathrm{kw}$ wind mill tower using different cross sectional areas. Author obtained optimum design trends through the use of the interior penalty function technique. Prowell [5] has determined the dynamic properties of 52 meter high $900 \mathrm{KW}$ wind turbine considering 3 different types of soil and has concluded that soft soil will influence dynamic properties of tower. In other research Prowell [6] has carried out the full scale wind turbine testing for $65 \mathrm{KW}$ having 22.6 $\mathrm{m}$ hub height. The different earthquake time histories are applied on the full scale turbines and he has observed degradation of grout at the tower base, and loss of bolt torque at the connections between tower segments. Harte [7] has studied the effects of modeling the soil and foundation for the wind turbines and he has studied the effect of soil in terms of displacement, base shear, shear force and bending moment in the turbine and foundation system. According to the Ministry of New and Renewable energy Government of India[8] total wind power potential of $302251 \mathrm{MW}$ has been estimated at 100 meter height In India, and only 21 percent us used by India. Hamaydeh [9] has modeled the wind turbine of 2 villages located in alsaka considering the soil properties prevailing at site. He has investigated the pile foundation for given site. Researcher has changed the dimensions of pile and spacing of pile and given the foundation design at two different sites. Kjorlaug[10] has modeled $65 \mathrm{KW}$ and $5 \mathrm{MW}$ 
wind turbine and applied wind and earthquake forces on the wind mill tower. He has also modeled soil at the foundation and has concluded that soil must be modeled to study the response of the wind mill tower. Jerath[11] has modeled 3 different wind mill turbines of $65 \mathrm{KW}, 1 \mathrm{MW}$ and $5 \mathrm{MW}$ capacity in the FEM software and performed dynamic analysis of the turbines and applied acceleration time history of 3 different earthquakes and studied the peak acceleration and deformation Reponses at various levels of the tower and has also concluded that the change in the damping ratio will not affect much more in the response in two horizontal directions but change in damping has significant effect on the vertical direction response. Bhattacharya [12] has studied the dynamic properties of off-shore wind turbine considering soil structure inter action and has concluded that the frequency of offshore turbines largely depends on the foundation type and soil type, Therefore in analysis of turbines the effects of soil must be considered for avoiding resonance conditions.

\section{FEM MODELING AND VALIDATION}

For validation in present work the wind mill data of work done by Prowell, [6] is considered.

In his research author has done the experimental work on the wind mill tower. The tower is $65 \mathrm{KW}$ tower and it consists of the three different hollow sections of the different diameters. The diameter is more in the bottom of the tower and to achieve the economy in the design the diameter is reduced at the top. Figure 1 shows the main parts of the wind mill structure. It shows the details of tower such as diameter of each section height of various sections. Various types of material are used in different components of the tower. The properties of material are shown in table 1. Based on these properties the tower is modeled in FEM software.

The tower was modeled using cylindrical shell property of steel and the nacelle of the tower was modeled as solid elements of a user-defined material with the correct mass.

\begin{tabular}{cccc} 
TABLE I: MATERIAL PROPERTIES \\
\hline \hline Component & Material & $\begin{array}{c}\text { Mass } \\
\text { Density } \\
\mathrm{kg} / \mathrm{m} 3\end{array}$ & $\begin{array}{c}\mathrm{E} \\
\mathrm{N} / \mathrm{mm} 2\end{array}$ \\
\hline Tower & Steel S275 & 9891 & 200000 \\
Nacelle & User & 1529 & 200000 \\
Rotor & Steel S275 & 1101 & 210000 \\
Blades & Polyester & 1101 & 1000 \\
\hline \hline
\end{tabular}

The blades of the given towers are modeled as cylindrical sections of fiber glass reinforced polyester material defined through the user-defined material In the FEM software. The turbine was assumed fixed at its base. The meshing of all the shell members of the tower has been done according to the requirements

The blade is properly connected with the axle and the axle is properly connected with the nacelle. In the FEM software Shell formulation will combine membrane and plate behavior and each joint within a shell object has six degree of freedom. Since joints within the frame objects also have six degrees of freedom, frames may connect directly to the joints of shell objects.
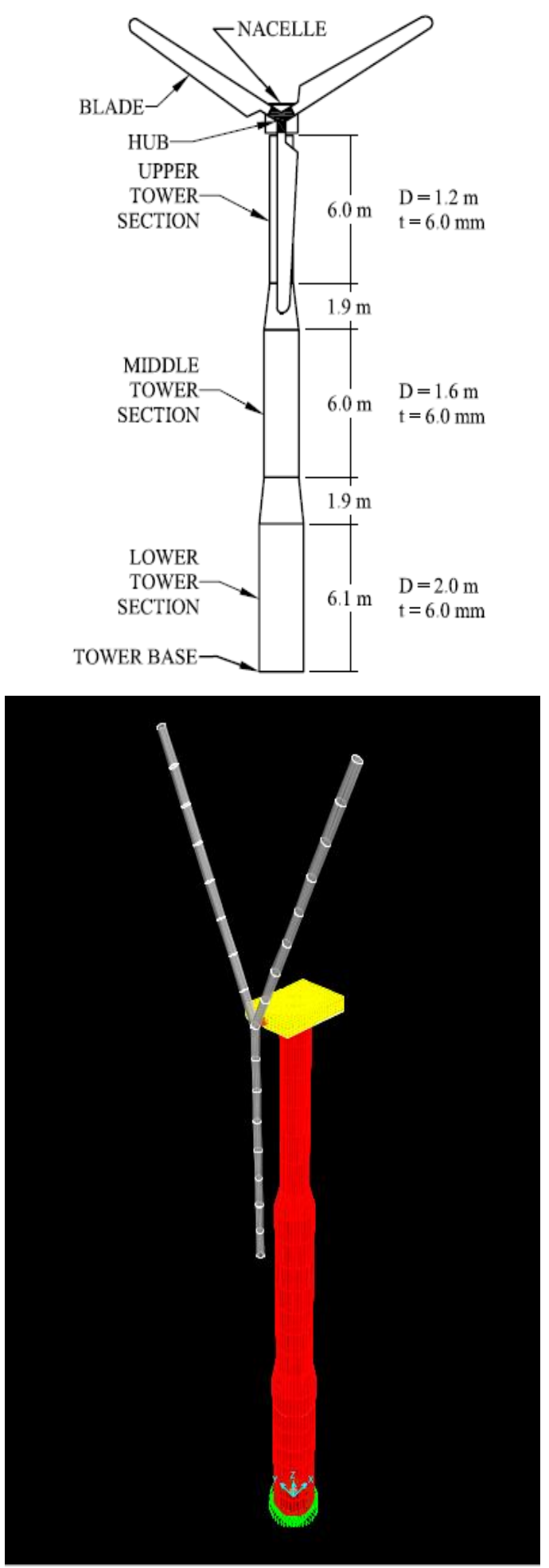

Fig 1. Details of tower and FEM model.

The blades are modeled as a frame element and it is connected with the solid. Joints within frame has six degrees of freedom but solid objects have only 3 translational degrees of freedom, Therefore, they provide no rotational resistance to interconnected frame and shell objects. A body constraint or rigid link should connect at the end joint of a frame to the tributary joints of a solid such that a force couple is available to resist moment within the frame joint, so for this proper structural behavior and connection between solid and shell objects a body constraint is also provided in FEM modeling to connect the solid and frame elements with each 
other

\section{A. Results of Model Validation}

The dynamic analysis of tower is carried out in the FEM software using Eigen value analysis. The number of modes are selected in such a way that modal mass participation ratio is more than 90 percentage, so that maximum mass of tower will participate in dynamic analysis. The frequency of the tower in various modes is obtained. The frequency of the tower in various modes is shown in the table 2 . We can observe that we are getting almost the same frequency in different modes according to earlier research

\begin{tabular}{|c|c|c|}
\hline Direction & $\begin{array}{c}\text { Frequency } \\
\text { by Prowell } \\
{[8]} \\
\mathbf{H z} \\
\end{array}$ & $\begin{array}{c}\text { Frequency } \\
\text { by present } \\
\text { work } \\
\mathbf{H z} \\
\end{array}$ \\
\hline Fore Aft & 1.70 & 1.86 \\
\hline Side to side & 1.71 & 1.86 \\
\hline Fore Aft & 11.9 & 11.00 \\
\hline Side to side & 12.4 & 11.59 \\
\hline
\end{tabular}

\section{DyNAMIC RESPONSE ANALYSIS OF THE MONOPOLE AND INNOVATIVE HYBRID TOWER CONSIDERING VARIOUS TYPES OF SOIL}

In order to study the influence of dynamic response in different site conditions, different types of soils are modeled in FEM software. In the previous work, the towers are modeled as monopoles having height of 22.0 meters. But as discussed earlier if the height of tower is increased we may get more wind at particular place and more power can be generated. But by increasing the height of tower the tower becomes dynamically sensitive and during operation conditions of turbine, the resonance condition may occur. In order to study the effect of increasing height of the monopole towers on dynamic properties of the soil monopole tower of 32 meter height is modeled in the FEM software as shown in fig.2. After studying the monopole tower an innovative hybrid tower which is combination of monopole tower and lattice tower is modeled. The height of Innovative hybrid tower is also considered 32 meters with bottom 17.4 meter is modeled as lattice tower and remaining 14.6 meter height is modeled as monopoles. In the innovative hybrid tower the bottom portion of tower is lattice tower and top portion is monopole. In FEM modeling main leg of lattice tower is modeled as beam element and the internal bracing systems are modeled as truss elements. For the analysis of the tower various loads such as dead load which includes self weight of the tower, nacelle, and blade are considered the FEM model of hybrid tower shown in Fig. 2.

It is not possible that wind mill towers are always constructed on hard soils, it may be constructed on medium or soft soil, so depending on different soil the dynamic properties of the tower such as natural frequency, time period of the tower may change. To study the effect of soil on dynamic properties of tower this monopole and innovative hybrid tower is modeled using 3 types of different soil

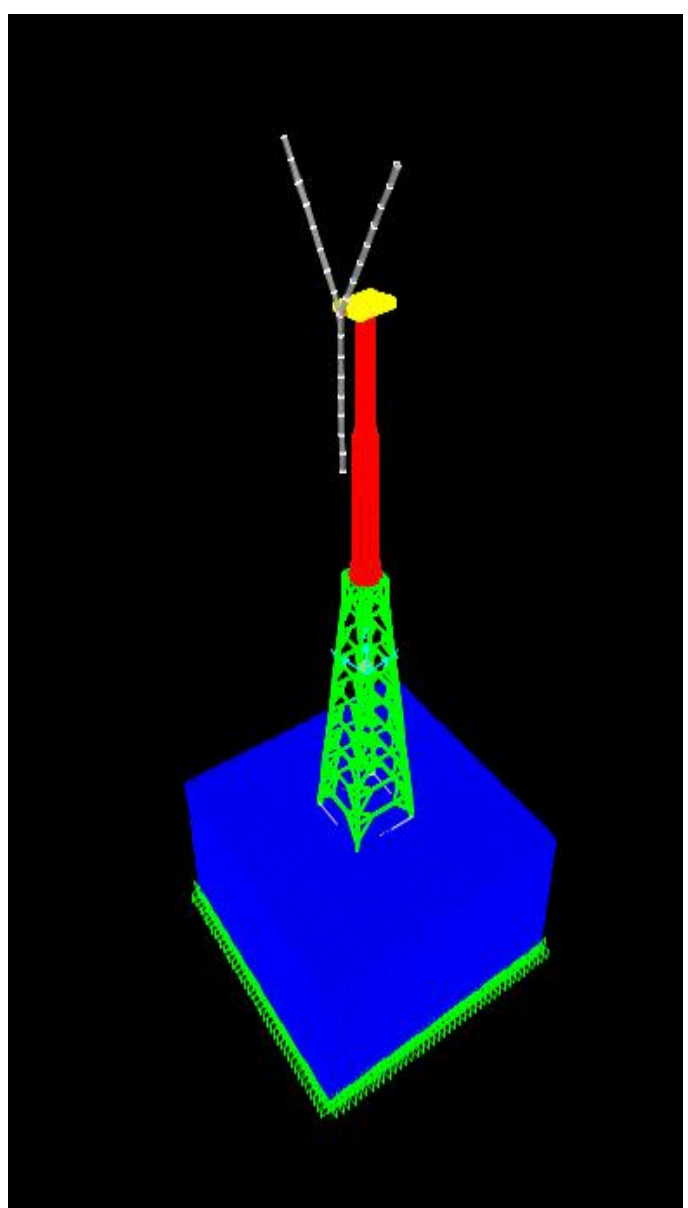

Fig. 2. FEM model of monopole tower with soil.

Condition. It is modeled considering tower on hard soil, medium soil and soft soil. The properties of the soil considered in FEM modeling are shown in Table III.

TABLE III: DYNAMIC PROPERTIES OF TOWER

\begin{tabular}{cccc}
\hline \hline Type of soil & $\begin{array}{c}(\mathbf{G}) \\
\mathbf{k n} / \mathbf{m} 2\end{array}$ & $\begin{array}{c}(\mathbf{E}) \\
\mathbf{k n} / \mathbf{m} 2\end{array}$ & $\begin{array}{c}\text { Poission's } \\
\text { ratio }\end{array}$ \\
\hline Hard & 30000 & 72000 & 0.20 \\
Medium & 20000 & 50000 & 0.25 \\
Soft & 10000 & 26000 & 0.30 \\
\hline \hline
\end{tabular}

After applying all the loads on the tower it is required to check the seismic capacity of monopole and innovative hybrid wind mill towers. So to study the effects of seismic forces on the tower the acceleration time history of the 2 major earthquakes such as Bhuj and Nepal are selected and time history is applied at the base of the tower and nonlinear dynamic analysis of the tower has been carried out.

\section{Modeling OF SOIL As FEM}

Soil is assumed to be an isotropic, homogeneous, linearly elastic. The behavior of such soil can be idealized and represented using solid elements in FEM software. For modeling the soil as solid element it is required to give shear modulus, elastic modulus and poisons ratio in the FEM software. The solid element is eight nodded element having three degrees of freedom of translation and rotation in the respective co ordinate directions at each node. 


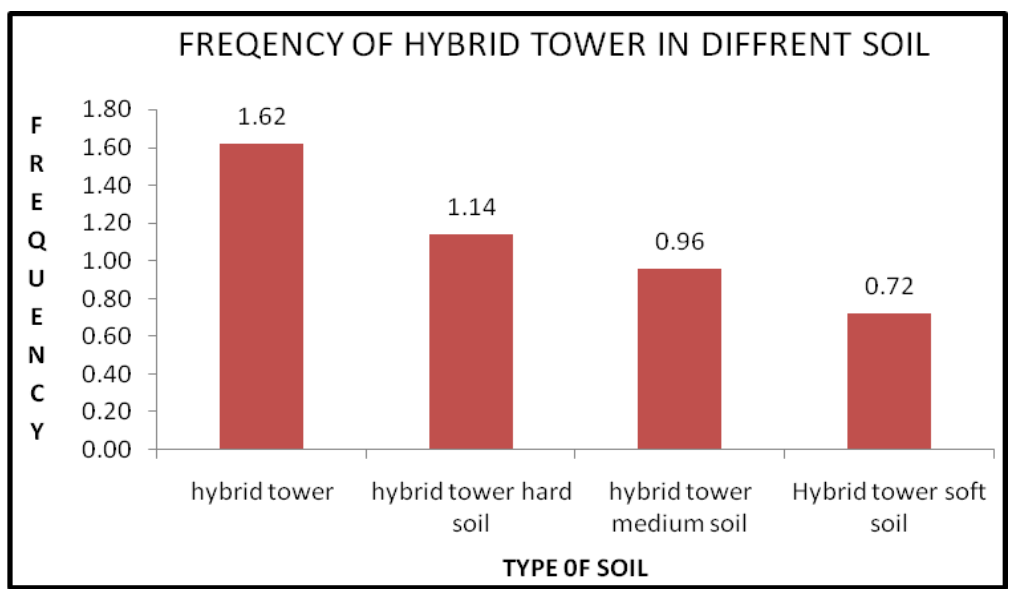

Fig. 3. Frequency of monopole tower with different soil.

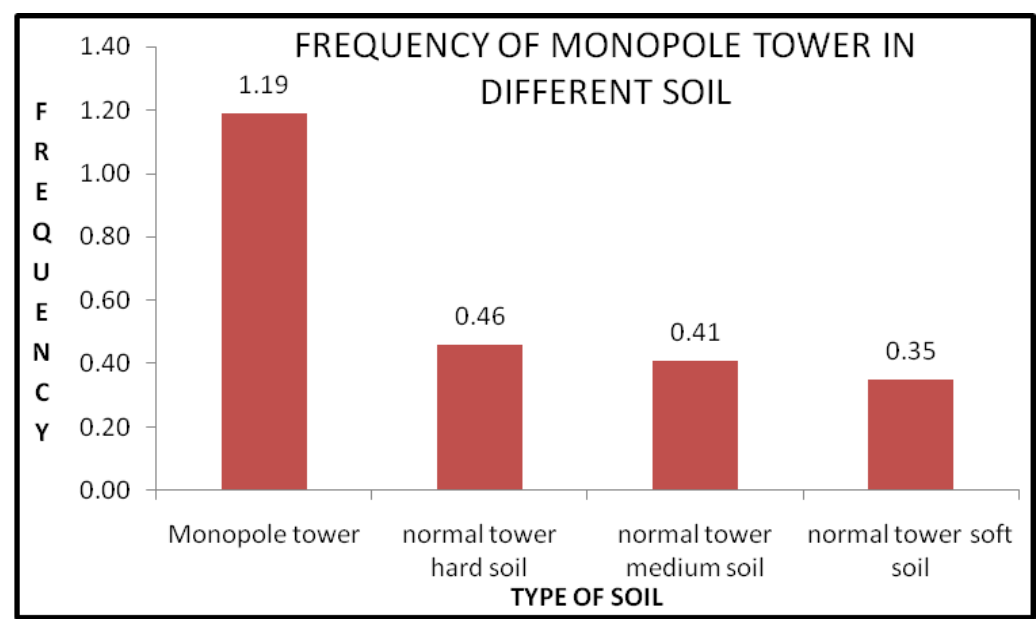

Fig. 4. Frequency of Hybrid tower with different soil.

Normally the width and depth of soil is kept in such a way that it affects the behavior of the superstructure. So to represent the soil as Continuum model it is represented by considering breadth equal to twice the width of the foundation along the plan dimension and thrice the width of foundation along the depth of foundation. So in present research work 20 m X $20 \mathrm{~m}$ X $10 \mathrm{~m}$ size soil is considered below tower. The FEM model of innovative hybrid tower including soil modeled at its base is shown in Figure. 2. The size of solid block is kept as $1 \mathrm{mt} \mathrm{X} 1 \mathrm{mt} \mathrm{X} 1 \mathrm{mt}$. the size of 8 nodded solid elements is selected in such a way that it resembles the actual soil condition of the site. The input of soil parameters are unit weight of soil, poission's ratio and modulus of elasticity of the soil. The soil behavior is assumed as linear and elastic material.

\section{DYNAMIC LOADS ON WIND TURBINE}

The wind mills are subjected to the dynamic loads due to vibrations and rotation of the blades which is fixed at the top of nacelle. Generally these dynamic loads are defined in terms of frequency and this frequency is known as $1 \mathrm{P}$ frequency. The other dynamic load on the tower is blade passing frequency, which is known as $2 \mathrm{P} / 3 \mathrm{P}$ frequencies. The blades of the wind turbine passing in front of the towers will cause a shadowing effect and produce a loss of wind load on the tower This is a dynamic load having frequency equal to three times the rotational frequency of the turbine $(3 \mathrm{P})$ for three blade wind turbines or two times $(2 \mathrm{P})$ the rotational frequency of the turbine for a two blade turbine. The $2 \mathrm{P} / 3 \mathrm{P}$ frequency of the turbine is simply obtained by multiplying the limits of the $1 \mathrm{P}$ frequency by the number of the turbine blades. In present case, the $1 \mathrm{p}$ frequency of the turbine under study is 45 to $55 \mathrm{RPM}$ so it is $0.75 \mathrm{~Hz}$ to $0.92 \mathrm{~Hz}$ and from this the $3 p$ frequency is $2.25 \mathrm{~Hz}$ to $2.76 \mathrm{~Hz}$.

The loads applied on the tower is the dynamic load due to blade rotation and self weight of tower, As we know that to avoid the resonance condition in the system, the designed frequency of overall system must be kept away from the frequency of applied loads. There are 3 possible ranges in which the natural frequency of the whole system may fall, which is soft- soft (natural frequency $<1 \mathrm{P}$ ), soft- stiff (Natural frequency between $1 \mathrm{P}$ and $3 \mathrm{P}$ ) and stiff-stiff (Natural frequency $>3 \mathrm{P}$ ). It is required to have accurate evaluation of natural frequency of tower; this natural frequency of system also depends on the stiffness of the foundation on which the towers are constructed. Fig. 3 shows the frequency of the 32 meter high tower considering stiffness of the soil for monopoles and Fig. 4 shows the frequency of the innovative hybrid tower considering stiffness of the soil.

Fig. 3 and Fig. 4 show the natural frequency of the tower considering simple support and 3 different types of the soil. The wind mill towers are the tall towers with heavy mass provided at the top of towers. So they are dynamically very. 


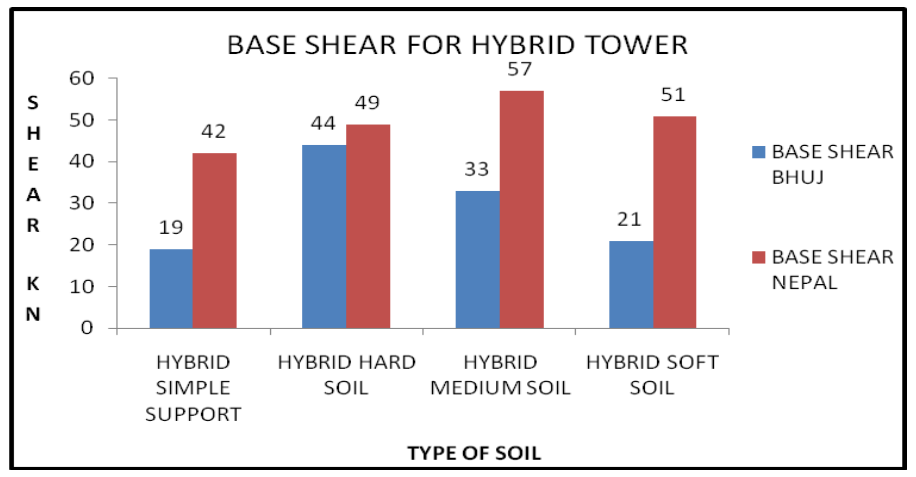

Fig. 5. Base shear for hybrid tower.

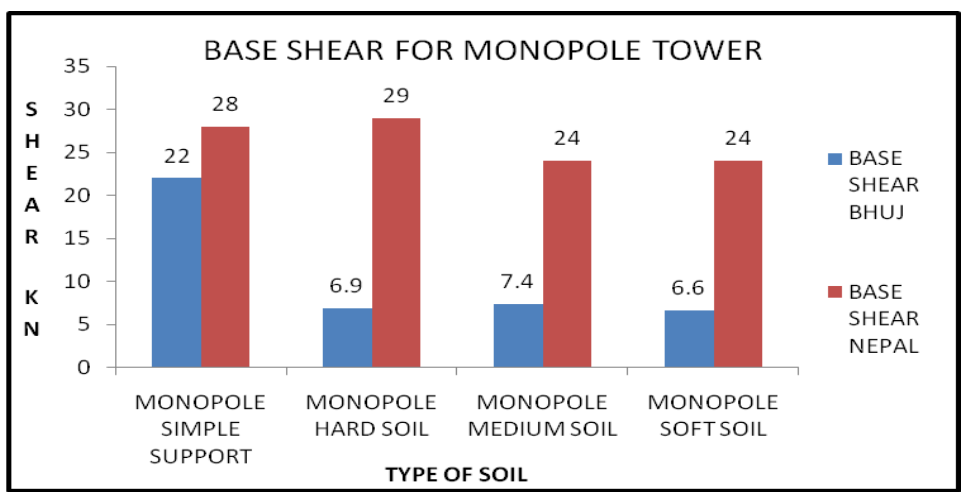

Fig. 6. Base Shear for Monopole Tower.

Sensitive systems. The natural period of tower system is affected by the soil on which towers are constructed. From fig.3, we can see that natural period of monopole tower is decreased by 240 percent compared to tower modeled without soil. So there is large variation in frequency of monopole tower. From fig.4, we can see that natural period of hybrid tower is decreased by only 55 percent compared to tower modeled without soil. So there is very less variation in frequency of hybrid tower compared to monopole tower.

Fig. 5 shows the base shear for the hybrid tower for different types of soil such as hard, medium and soft soil. As the Nepal earthquake was very severe compared to the Bhuj earthquake we get higher value of base shear. The base shear also depends on the type of soil and the type of the tower. We get maximum value of base shear for Nepal earthquakes in the medium soil and higher value of base shear for the hard soil in the Bhuj earthquake. So we can observe that base shear also varies for different types of the soil.

Fig. 5 shows the base shear for the hybrid tower for different types of soil such as hard, medium and soft soil. As the Nepal earthquake was very severe compared to the Bhuj earthquake we get higher value of base shear. The base shear also depends on the type of soil and the type of the tower. We get maximum value of base shear for Nepal earthquakes in the medium soil and higher value of base shear for the hard soil in the Bhuj earthquake. So we can observe that base shear also varies for different types of the soil.

Fig. 6 shows the base shear for the monopole tower for different types of soil we get higher base shear in hard soil for Nepal earthquake and higher value of base shear for the bhuj earthquake.

By comparing the base shear of monopole and hybrid towers we get higher base shear in the hybrid towers because the mass of the hybrid tower is higher compared to the monopole towers. As well as hybrid towers are stiff towers compared to the monopole towers so we get higher base shear but on the other hand the variation of frequency for different soil conditions are less in hybrid tower compared to monopoles.

So hybrid towers can be effectively constructed in the area where soil conditions are soft. As the variation in frequency of tower considering soil is less there are less chances of resonance condition in the hybrid towers.

\section{CONCLUSION}

From the above study we can conclude that

- The effect of the soil is more predominant in case of tall wind mill structures constructed on the soft soil and it can result in resonance conditions during the operation of wind mill towers so stiffness of soil must be considered for dynamic analysis of tower.

- By increasing the height of monopole tower it results in the resonance conditions for different types of soil, but due to less variation in the natural frequency of the innovative hybrid tower it will eliminate the resonance conditions during operations of turbine.

- Innovative hybrid towers are less sensitive to dynamic loads and can be used very efficiently for tall wind mill structures.

\section{REFERENCES}

[1] B. Gencturk, A. Attar, and C. Tort, "Design optimization of lattice wind turbine towers subjected to earthquake forces structures congress ASCE," 2014. 
[2] B. Song and J. C. Wu, "Comparative analysis on dynamic response of wind turbine tower in different sites international efforts in lifeline earthquake engineering ASCE, 2014.

[3] D. Lombardi, S. Bhattacharya, and D. M. Wood, "Dynamic soil-structure interaction of monopile supported wind turbines in cohesive soil," Soil Dynamics and Earthquake Engineering, vol. 49, 2013.

[4] H. M. Negma and K.Y. Maalawi, "Structural design optimization of wind turbine towers computers and structures," Elsevier Science Ltd. vol.74, 2000

[5] I. Prowell, A. Elgamal, J. Lu, and J. E. Luco, "Modal properties of a modern wind turbine including," in Proc. the 17th International Conference on Soil Mechanics and Geotechnical Engineering, 2009.

[6] I. P. E. Prowell, M. ASCE, C. M. M. Uang, M. J. A. Elgamal, E. Luco, and L. Guo, "Shake table testing of a utility-scale wind turbine," Journal of Engineering Mechanics ASCE, 2012, pp. 900-909.

[7] M. Harte, B. Basu, and S. R. K. Nielsen, "Dynamic analysis of wind turbines including soil-structure interaction engineering structures," vol. 45, 2012.

[8] Ministry of New and Renewable Energy, Government of India website. [Online]. Available: http://www.mnre.gov.in

[9] M. A. Hamaydeh and S. Hussain, "Optimized frequency-based foundation design for wind turbine towers utilizing soil-structure interaction," Science Direct Journal of the Franklin Institute, vol. 348 , 2011, pp. 1470-1487

[10] R. A. Kjørlaug, A. M. Kaynia, and A. Elgamal, "Seismic response of wind turbines due to earthquake and wind loading," in Proc. the 9th International Conference on Structural Dynamics, 2014.

[11] S. Jerath and S. Austin, "Response of wind turbine towers to seismic loading at different damping ratios structures congress," 2013.
[12] S. Bhattacharya and J. A. Cox, "Domenico lombardi david muir wood dynamics of offshore wind turbines supported on two foundations ice proceedings," Geotechnical Engineering, vol.166, 2012.

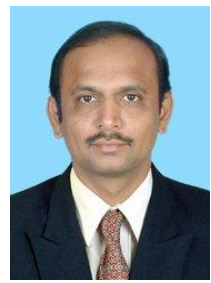

Hemal J. Shah has done his graduation in 2002 and masters in structure in 2013 from Sardar Valaabhbhai National Institute Of Technology Surat. He is working as assistant professor in Applied Mechanics Department at Government Engineering College, Bharuch, Gujarat, india. He is pursuing $\mathrm{PhD}$ from Sardar Valaabhbhai National Institute Of Technology Surat.

He has teaching experience of 13 years and guided post graduate students in structural field. He has published 10 research papers in international journals. His area of interest is analysis of tall structures, dynamic analysis of structures and seismic analysis of structures.

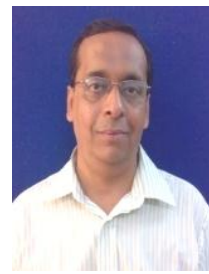

Atul K. Desai has done his graduation in 1983 and masters in structure in 1985 from Sardar Valaabhbhai National Institute Of Technology Surat. He has done Phd in 2008 from Sardar Valaabhbhai National Institute of Technology Surat $\mathrm{He}$ is working as professor in Applied Mechanics Department at Sardar Valaabhbhai National Institute Of Technology Surat. Gujarat India.

He has teaching experience of 34 years and guided $11 \mathrm{PhD}$ students and 70 post graduate students in structural field. He has published more than 100 research papers in reputed international journals. His area of interest is Structural Dynamics; Machine Foundations; Bridge Engineering; Earthquake Engineering and Structural Forensic 